\title{
Die Galgen von Mergentheim
}

\section{Zur Geschichte des Hochgerichts in Mergentheim}

\author{
von Alice Ehrmann-Pösch
}

\section{Galgen als Zeichen der Blutgerichtsbarkeit}

Der Galgen, in alten Karten als Hochgericht eingezeichnet, lag weithin sichtbar in der Nähe der großen Reichs- und Handelsstraße nach Nürnberg und Augsburg, unterhalb des heutigen Spessartblicks. Nicht nur die Aussicht ist bis heute von hier aus bemerkenswert; man wurde auch weithin gesehen. Ein solches Hochgericht diente zunächst einmal für jeden Vorbeikommenden als Abschreckung, war aber daneben auch eine praktische Kennzeichnung des jeweiligen Herrschafts-, bzw. Gerichtsbezirks: Man betrat nun als Reisender ein neues Territorium mit anderem Fürsten, bzw. anderem Gerichtsherren. Ein Markierungszeichen, das in dem in einzelne kleine und kleinste Fürstentümer zersplitterten Deutschen Reich zur Orientierung der jeweiligen Territorien diente.

Das mit einem komplizierten und umfangreichen Zoll-, Geleit- und Wegerechtssystem ausgestattete Verkehrsnetz beschwerte eine Reise. Als Hinweis auf einen Wechsel des Territoriums und damit einhergehend neu entstehende Kosten konnte ein Galgen eine Orientierungshilfe darstellen. Jeder Galgen wurde daher ziemlich nahe an der Grenzmarke errichtet. Dabei musste aber unbedingt beachtet werden, dass der Schatten des Galgens nicht auf das angrenzende Gebiet fallen durfte, denn das wäre als Ausdehnung der Jurisdiktion auf nachbarschaftliches Gebiet und damit als Provokation gewertet worden ${ }^{1}$. Andererseits sollten die Galgen auch in entsprechendem Abstand zur Straße errichtet werden daß

1 Johann Georg Krünitz: Oekonomisch-technologische Enzyklopädie. Bd. 15. Berlin ${ }^{2} 1786$, S. 673. Vgl. aus der neueren Literatur auch grundlegend: Jutta Novosadtko: Scharfrichter und Abdecker. Der Alltag zweier „unehrlicher Berufe“ in der Frühen Neuzeit. Paderborn u. a. 1994, S. 11, 83 f., 95, 192 f., 304. Gerhard Schwarz: Die Ulmer Scharfrichter nach den Ratsprotokollen. In: Südwestdeutsche Blätter für Familien- und Wappenkunde 14 (1974), S. 24-28; Wolfgang Scheffknecht: Scharfrichter. Eine Randgruppe im frühneuzeitlichen Vorarlberg. Konstanz 1995. Vgl. im Übrigen die umfangreiche überregionale und regionale Literatur zur Todesstrafe bei Gerhard Fritz: Von der öffentlichen zur nichtöffentlichen Vollstreckung. Zur Todesstrafe im Königreich Württemberg 1818 bis 1871. In: Gerhard Fritz / Daniel Kirn (Hrsg.): Florilegium Suevicum. Beiträge zur südwestdeutschen Landeskunde. Festschrift für Franz Quarthal zum 65. Geburtstag. Ostfildern 2008 (= Stuttgarter historische Studien zur Landes- und Wirtschaftsgeschichte 12), S. 245-274, hier 265. 


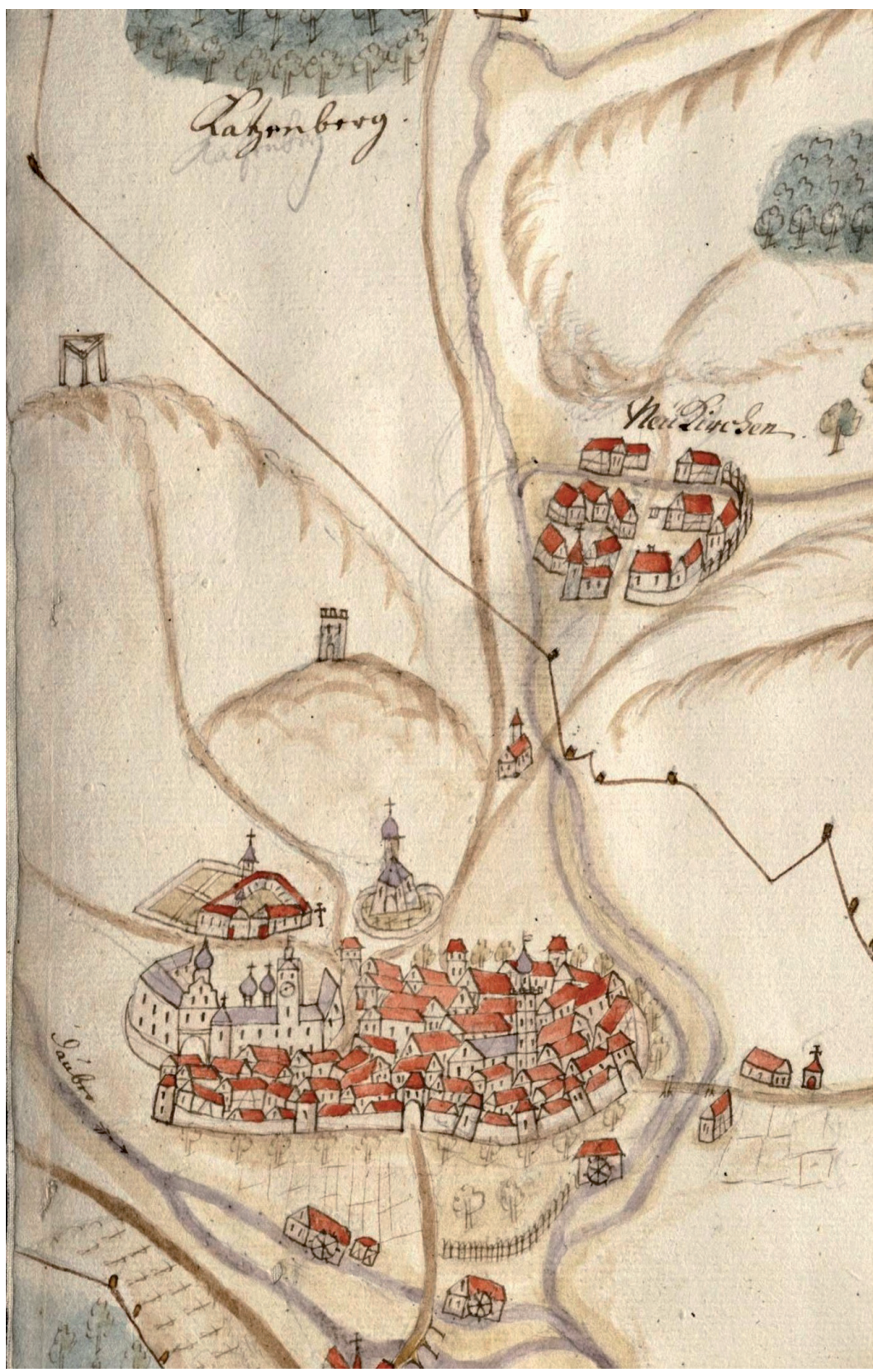

Abb. 1: Markungsatlas der Deutschordensregierung Mergentheim, kolorierte Feder- und Pinselzeichnung (Vorlage und Aufnahme: HStA Stuttgart, N 13 Nr. 22, Bl. 3, Ausschnitt) 
man sie zwar deutlich erkennen könne, jedoch auch also weit damit ihr Anblick und übler Geruch der Gesundheit der Reisenden nicht nachtheilig werde ${ }^{2}$.

Der Galgen als „,repräsentativer“ Bau bezeugt auch die Häufigkeit der Durchsetzung von Recht und Ordnung. Ein heruntergekommener Galgen wurde demgemäß eher mit einer schwachen oder laxen Handhabung des Rechts gleichgesetzt. In der Enzyklopädie von Krünitz aus dem Jahr 1786 steht hierzu: Man lieset von dem Kaiser Maximilian dem I, daß, wie er einst vor einen Galgen vorbey gereiset sey, er sein Haupt entblößet, und gesaget habe: Salve Justitia! Eben diese wird auch von einem Könige in Frankreich erzählet, daß er allemahl, wenn er vor einen Galgen vorbey gereiset sey, den Hut abgezogen, sich geneiget und ihm gedankt habe, mit Vermelden, dieser erhielte ihm mehr als der Scepter, den er in Händen führete ${ }^{3}$.

\section{Frühe Nachrichten vom Mergentheimer Galgen}

In Mergentheim und vielen benachbarten Dörfern war der Deutsche Orden seit 1340 nicht nur Stadt-, sondern auch Landesherr über ein kleines Territorium, in dem er folglich auch oberster Gerichtsherr war. Damit verbunden war die Ausübung der Blutgerichtsbarkeit - das Recht, über Leben und Tod zu urteilen. Es ist daher davon auszugehen, dass bereits zu jener Zeit ein Galgen an diesem Ort vorhanden war. Spätestens seit dem frühen 15. Jahrhundert ist ein Henker hier am Ort nachweisbar ${ }^{4}$, der den Galgen als Ort des Rechtsvollzugs bediente, denn in der Wimpfener Rechtsweisung für Mergentheim heißt es 1426, dass ein Dieb hinausgeführt werden und am Hochgericht seine gerechte Strafe erhalten sollte ${ }^{5}$. Erste Nachrichten über den Galgen bekommen wir aus dem Jahr 15376. Der Galgen musste erneuert werden und dabei waren viele Zeremonien nötig (hierzu später). Breitenbach - ein Mergentheimer Chronist des frühen 19. Jahrhunderts - berichtet, dass der Galgen bereits einige Tage nach dessen Aufrichtung 1537 benötigt wurde, denn Hans Kuhn brachte einen Crailsheimer namens Leonhardt Holzer wegen Diebstahls vor Gericht, welches des Holzers noch am nemlichen Tage aufhängen ließ $\beta^{7}$.

\footnotetext{
2 Krünitz (wie Anm. 1), S. 674.

3 Ebd., S. 673.

4 Wimpfener Rechtsweisung für die Stadt vom 4. April 1426, Deutsches Rechtswörterbuch (http:// drw-www.adw.uni-heidelberg.de/drw/), Rechtstexte als Quellen, Oberrheinische Stadtrechte I, 1895, S. 155.

5 Erwähnt auch bei Franz Diehm: Geschichte der Stadt Bad Mergentheim. Äußeres Schicksal und innere Verhältnisse. Bad Mergentheim 1963, S. 104.

6 Paul Anton Breitenbachs Chronik, 59v, um 1834, handschriftlich im Stadtarchiv Mergentheim.

7 Breitenbach (wie Anm. 6) Bl. 119, bzw. 60r, genauere Angabe bei Adolf Renz: Der Scharfrichter im alten Mergentheim, Tauber-Zeitung 29.12.1936 mit der Angabe Freitag nach Sebastiani (20. Januar).
} 


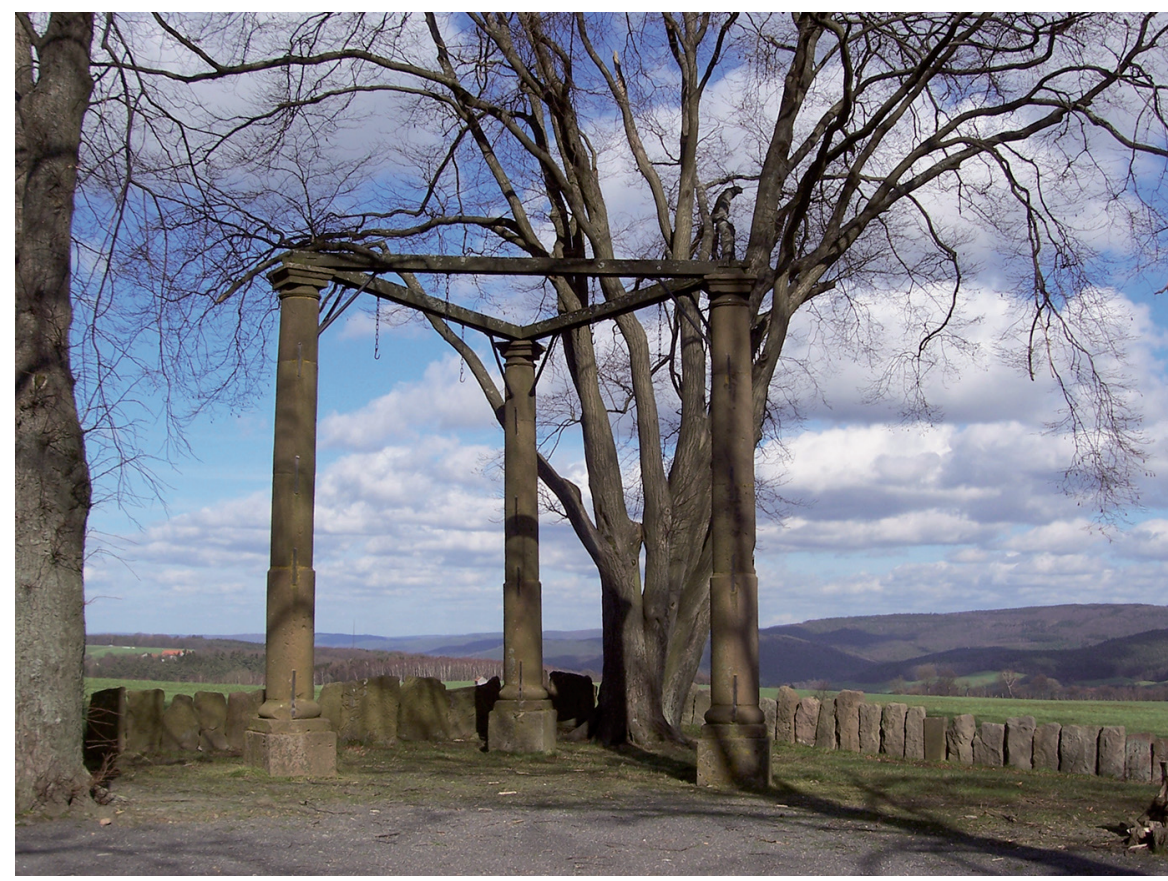

Abb. 2: Der Galgen von Beerfelden in der Nähe von Michelstadt im Odenwald

1574 war der Galgen wieder morsch. Man entschloss sich nun, einen neuen Galgen mit drei steinernen Säulen auf dem Kitzberg ${ }^{8}$ zu errichten, der auch, immer wieder repariert, bis ins 19. Jahrhundert im Grunde unverändert blieb. Es handelte sich um einen dreischläfrigen Galgen. Die steinernen Säulen waren zu einem Dreieck angeordnet und mit quer liegenden Balken verbunden, so dass drei Balken mit Delinquenten ,bestückt“" werden konnten, daher dreischläfrig”. Auf vielen Flur- und Gemarkungskarten wurde er so eingezeichnet, jedoch nur sehr summarisch, so dass man keine Details daraus ablesen kann.

Die nächste überlieferte Renovierung erfolgte 1628. Dies war in Mergentheim und vielen anderen Orten eine Zeit, in der die Strafjustiz auf Hochtouren lief - es war die Zeit der großen Hexenverfolgungen. Ob die Renovierung des Hochgerichtes damit in unmittelbarem Zusammenhang stand, kann nicht genau nachgewiesen werden. Man nimmt an, dass die Hexenverbrennungen eher nicht am Hochgericht, sondern auf dem Tauberwasen bei der Wolfgangsbrücke stattgefunden haben, damit man die „unreine“ Asche in die Tauber entsorgen konnte.

8 Ratsbuch von 1574 für 188 fl nach Adolf Renz (wie Anm. 7), Breitenbach (wie Anm. 6), Bl. 71v. 9 Vgl. die zahlreichen Abbildungen von Galgen bei Wolfgang Schild: Alte Gerichtsbarkeit. Vom Gottesurteil bis zum Beginn der modernen Rechtsprechung. München ${ }^{2} 1985$, S. 63-67. 


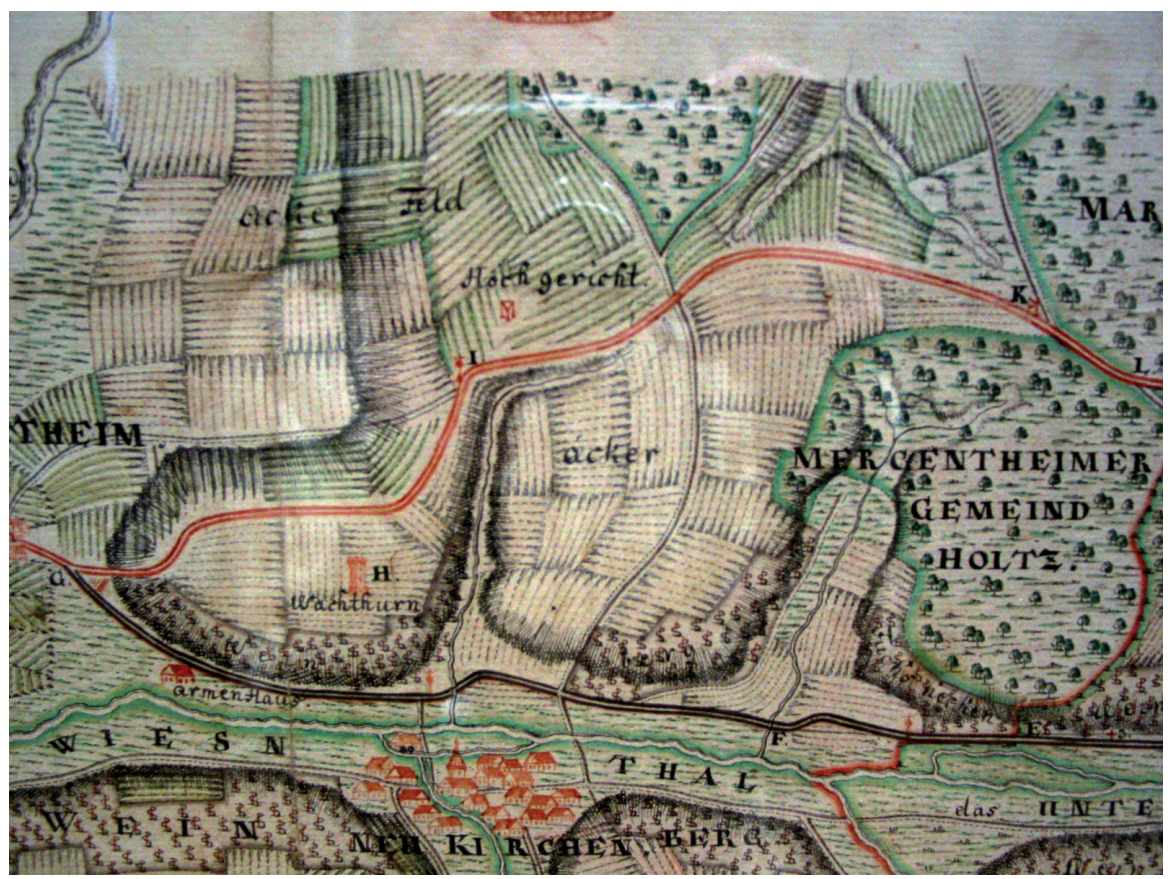

Abb. 3: Karte von Matthias Kohler, kolorierte Federzeichnung, 1777

(Deutschordensmuseum Inv. Nr. 2539).

Über die Renovierung am Hochgericht hat sich allerdings nur eine Unkostenrechnung des Althäuser Boten wegen ufgebauten newen Hochgerichts ${ }^{10}$ erhalten, Ebenso eine Rechnung von $1632^{11}$ über Balken, Eisen, Nägel und Strang für das Hochgericht.

Aus dem Jahr 1630 sind wieder zwei Hinrichtungen überliefert, die nichts mit Hexerei zu tun hatten. Der eine Delinquent war Kilian Haan ${ }^{12}$, der dem Zimmerwarter im Schloss 700 fl, eine gewaltige Summe, gestohlen hatte und daher zum Strang verurteilt wurde, der andere war der Obermanger ${ }^{13}$, der zum Schwert verurteilt wurde, ohne dass man hierüber aus den Akten die näheren Gründe erfährt.

10 StAL B 246 Bü 220.

11 StadtA Mergentheim, Bürgermeisteramtsrechnungen von 1632.

12 StadtA Mergentheim F 3011.

13 Ebd. 


\section{Provisorische Reparatur des Galgens 1711 und 1712 Pläne für eine gründliche Reparatur 1712}

Eine größere Aktion fand im Zusammenhang mit der Hinrichtung des Hans Georg Rothaupt aus Rengershausen am 10. Dezember 1711 statt $^{14}$. Der gewesene Schulmeister Rothaupt wurde wegen Einbruchs in die Hofkapelle und Diebstahls von Altargerät zum Tode durch das Schwert und anschließender Verbrennung verurteilt. Aufgrund dieser nun notwendig gewordenen Reparatur konnte erst eine vorschriftsmäßige Hinrichtung gewährleistet werden. Zu diesem Zweck erging am 7. Dezember 1711 von der Regierung ein Befehl an alle Zimmerleute, Maurer, Wagner und Schmiede von allen Orten, die der Cent (= Gerichtsbezirk) Mergentheim unterstanden, am selben Nachmittag sich zu treffen, um die nötige Reparatur des ruinösen Hochgerichts vorzunehmen ${ }^{15}$.

Dieser Termin, der sehr kurzfristig angesetzt war, konnte nur die allernotwendigste Herrichtung ${ }^{16}$ bedeuten, da ja schon drei Tage später für die Hinrichtung alles bereit sein musste. Es wird über die benötigte Anlieferung von 3 Stamm Holtzes a 25 Schuh lang ${ }^{17}$, sowie von Kalk, Sand und Stein gesprochen. Das Baumaterial wurde auch für die Errichtung eines Schafotts verwendet, auf dem die Enthauptung stattfinden sollte. Neben den oben genannten Handwerkern waren die Müller von Mergentheim zur Anlieferung des Holzes verpflichtet: Es wurden zwei Fuhren Bauholz, sowie zwei Karren Speis zum Hochgericht befördert. Des Weiteren mussten die Müller das Brennholz und das Stroh für den decapitirten Cörper herbey und auf die Mahl- und Richtstatt ${ }^{18}$ schaffen, damit Rothaupt dort verbrannt werden konnte. Trotz Protestes mussten die Müller dem Befehl nachkommen. Für all dies wurden $12 \mathrm{fl} 35 \mathrm{x}$ in Rechnung gestellt ${ }^{19}$. Vom 13. April 1712 hat sich ein Schreiben an die Regierung erhalten, in dem

14 Siehe dazu: Heinrich Schmitt: Aus Mergentheimer Centgerichtsakten von 1711. In: Veröffentlichungen des Altertumsvereins Mergentheim 1893/94, S. 8 ff.

15 StadtA Mergentheim F 3012.

16 StAL B 246 Bü 220, Bericht Molitors vom 20. Mai 1712. Franz Simon Molitor war ab 1693 im Dienst des Deutschen Ordens in Mergentheim. Zunächst war er Registrator, dann Hofrat und Archivar.

17 StadtA F 3012, Dekret an die Zimmerleute vom 7. Dezember 1711.

18 StAL B 246 Bü 220, Molitorbericht vom 20. Mai 1712. In einer ausführlichen Stellungnahme geht Molitor auf die Heranziehung der Müller für Arbeiten am Hochgericht ein. Er erwähnt die Beschwerden der Müller deswegen und versucht die Aufgabenzuweisung zunächst pragmatisch zu erklären: Die Müller haben Roß und Geschirr. Etwas weiter unten bringt er aber doch den Berufsstand ins Spiel, denn Müller stehen fast aller Orten in diebischem Verdacht. Das Müllerhandwerk gehört zu den unehrenhaften Berufen. Die Lage der Mühlen außerhalb der Siedlungen und damit außerhalb der sozialen Kontrolle machte die Müller suspekt. Molitor übernimmt diese Auffassung aber nicht unkritisch, sondern beruft sich auf die Literatur um die Verpflichtung der Müller zu begründen und nicht auf die Tradition. Die Normen für unehrenhafte Berufe wurden im 18. Jahrhundert zunehmend aufgeweicht, daher sollte man eine Vorgabe, die auf diese Strukturen gründet, nun besser auf wissenschaftliche Autoritäten stützen, als auf das alte Herkommen.

19 StadtA Mergentheim F 3012. 
sich die Stadt bitter über die entstandenen Unkosten beschwert, da im Dezember 1711 doch nur der Fuß eines der drei Pilaren (Säulen) repariert worden sei, aber die Verköstigung der Maurer und Zimmerleute so teuer gekommen sei wie es der vollkommenen Reparation des gesamten Galgens entspräche. Ziemlich frustriert erwähnte der Schreiber, dass auch den zur Anwesenheit verpflichteten Vögten und Schultheißen ebenfalls eine gute Zeche gewährt wurde, während den Gerichtspersonen wie dem vorsitzenden Richter und den 12 Schöffen keine Zehrung erstattet würde: welche dem gantzo weret von Anfang biß End abwahrten und mit schwehren Pflichten sich belegen lassen müssen.

Dieser notdürftigen Herrichtung des Galgens sollte im Frühsommer 1712 eine grundlegende Erneuerung folgen. Aufgrund einer Abrechnung ist für den 13. und 17.Mai ersichtlich, dass der Scharfrichter zunächst die alten verfaulten Balken herab geworfen und weggeführt hat ${ }^{20}$.Die Maurer waren am 13. Mai am Hochgericht tätig (Zeche beim Straußenwirt von 7 fl 50 x), ebenso die Schmiede am 17. (Straußenwirt 6 fl 40 x), die Müller lieferten Stämme, Stein, Kalk und Gerüststangen an (Zeche $5 \mathrm{fl} 30 \mathrm{x}$ ).

Das heißt, die ersten Arbeiten waren das Aufschlagen des Gerüstes sowie die Anlieferung von Steinen, Sand und Kalk. Die Schmiede stellten die eisernen Klammern für das Mauerwerk, bzw. die Befestigung der Balken her. Schwierig wurde es, als die Querbalken, von denen jeder 21 Schuh (etwa 6,5 m) lang war, hinaufgezogen werden mussten, denn dazu waren neben den Zimmerleuten mindestens 30 Handlanger von Nöten ${ }^{21}$.

\section{Die Galgenreparatur 1712: Unwillige Handwerker}

Wer beteiligt sich schon gerne am Bau eines Galgens? Wie sollte man die Reparatur mit all den Handwerkern organisieren und nach welchen Regeln läuft das üblicherweise ab? Der Stadtschreiber wandte sich an das Archiv, um sich zu erkundigen, wie das Procedere von alters her war. Im Archiv ließ sich jedoch nichts auffinden. Mit den entschuldigenden Worten des Franz Simon Molitors alldieweilen von dergleichen zufälligen Begebenheiten niemahlen einig förmbliche Relationen mit schriftliche Berichte etwan verfasset, sollte jedoch nun schriftlich festgehalten werden, wie man die Handwerker auswählt, wer als Handlanger fungieren muss, und wer alles erscheinen muss. Darüber hinaus sollte ein schriftliches Verzeichnis aller ansässigen Centuntertanen, also aller Untertanen, die im Gerichtsbezirk ansässig waren, angefertigt werden. Er erwägt in diesem Schreiben, ob nicht die Handwerker eine Auswahl unter sich treffen sollten, oder ob die Herrschaft eine bestimmte Zahl anfordern solle, oder das gesamte Handwerk Hand anlegen sollte, wie es die Zimmerleute bei der Errichtung des Solda-

20 StadtA Mergentheim F 3012: 1 fl 15 x.

21 Ebd. und StAL B 246 Bü 220: Brief der Stadtschreiberei vom 11. Mai 1712 an die Regierung. 
tengalgens 1710 auf ihr in seinen Augen übel anführendes Altes Herkommenso Molitor - verlangten. Die Handlanger betreffend solle man diese, wie an anderen Orten auch üblich, aus der großen Bevölkerungsgruppe der Häcker oder der Müller oder der Leinweber nehmen.

Der Galgenbezirk ist anrïchig. Ein Hinrichtungsort ist ein zwielichtiger Ort, an dem man sich nur ungern aufhielt; man wusste nicht, welche dunklen Mächte hier vorhanden waren. Daher wollte niemand dort Arbeiten ausführen. Außerdem: Wenn nur ein einzelner Handwerker oder wenige Handwerker am Hinrichtungsort etwas zu reparieren oder zu bauen hatten, bestand die Gefahr, dass diese „unrein“ und nicht mehr von den Mitbürgern akzeptiert wurden. Deshalb war es eine weit verbreitete Praxis, möglichst viele, optimalerweise alle Handwerker eines Ortes bei den Reparatur- und Vorbereitungsarbeiten einzusetzen. So wurden von der Stadt die hiesigen Häcker (= Winzer) zusammengerufen und vor die Alternative gestellt, entweder zehn Freiwillige zu stellen, die dann um den gebräuchlichen Tageslohn die Arbeiten am Hochgerüst unterstützten, oder die Herrschaft bestimmte die Arbeiter ${ }^{22}$. In anderen Centbezirken wie in Ellwangen $^{23}$ war es üblich, dass Arbeiter, die am Hochgericht tätig werden mussten, den doppelten Lohn für diese unehrenhafte Arbeit als Ausgleich bekamen. Daher wundert die immer wiederkehrende Wiederholung umb einen gewohnlichen Taglohn nicht, für den die Handwerker in Mergentheim arbeiten mussten. Doch auch für den doppelten Taglohn wollten die Häcker als Handlanger nicht am Galgenbau tätig sein ${ }^{24}$.

Bezüglich des Extralohns, mit dem manche Gerichte die Arbeit am Galgen versüßen wollten, zeigte man sich in Mergentheim nicht zugänglich, denn es gab keine rechtlichen Grundlagen dafür. In vielen Gesetzeswerken, angefangen bei der Carolina (Strafgesetzbuch Karls V. von 1532, in § 215), ${ }^{25}$ wie in späteren Gerichts- und Gebührenordnungen (so in der für Mergentheim 1732 erlassenen Centtaxordnung Clemens Augusts) wird ausdrücklich betont, dass die Handwerker für diese ihnen unangenehme Arbeit keinen doppelten Taglohn erheben durften. Überhaupt war die Auswahl von zehn angeforderten Häckern problematisch, denn wer erklärte sich freiwillig aus ihrer Schar dazu bereit? Man hatte Angst um seinen guten Leumund. Bei den Alten sei es nicht so schlimm, begründeten dies die Häcker in einem Schreiben an den Bürgermeister, aber die Jungen müssten ihr ganzes Leben mit diesem vermeintlichen Makel leben.

22 Ausgenommen sein sollte die Jugend, StadtA Mergentheim F 3012.

23 Vgl. dazu Jaroslaw Piech: „,mit dem Strang zum Todt hingericht“. Der Ellwanger Galgen und andere Galgenstandorte in Württemberg. In: Fundberichte aus Baden-Württemberg 30 (2009), S. 512-755; ders.: Mit fliegenden Fahnen zogen sie zum Hochgericht. Der Galgen von Ellwangen an der Jagst 1701-1811, (in: Jost Auler (Hg.): Richtstättenarchäologie. Bd. 1), Dormagen 2008, S. 230-248.

24 StadtA Mergentheim F 3012.

25 Die Peinliche Halsgerichtsordnung Kaiser Karls V. von 1532 (Carolina). Hg. u. erläutert von Gustav Radbruch. 6. durchgesehene Auflage, hg. von Arthur Kaufmann. Stuttgart 1975 (= RUB 2990), §215, S. $127 \mathrm{f}$. 
Es gab auch die Möglichkeit, das Los entscheiden zu lassen, doch auch dies wäre keine befriedigende Lösung gewesen. Daher schalteten die Häcker den Bürgermeister Stellwaag ein und baten ihn um eine Eingabe an die Regierung, damit diese ihnen wie auch den Handwerkern mit einem gemessenen Decret [.. .] diese Handahnlegung ihro ahn hergebrachten guten Leymuth und Reputation zu keiner Zeit prajudicierlich versehen seyn und dies somit zu gewährleisten ${ }^{26}$. Der gute Ruf und die gesellschaftliche Reputation konnten in den Augen der Zeitgenossen durch solche Arbeiten ernsthaft gefährdet sein.

Da man von der Vorstellung ausging, dass ein anrüchiger, unehrenhafter Ort auch die Person, die damit zu tun hatte, anrüchig machte, sprich: dies mit dem Ehrverlust einherging, musste die Arbeit am Hochgericht durch strenge Zeremonien geregelt werden, die einem Ehrverlust vorbeugten. Die Errichtung oder Reparatur eines Galgens war daher keine rein handwerkliche Arbeit, sondern immer auch eine rituelle Tätigkeit, die mit vielen symbolischen Handlungen verbunden war. Die gesamte Gemeinde und alle, die zu diesem Centgebiet gehörenden Centverwandten wurden mit einbezogen.

Molitor schreibt in seinem Bericht vom 20. Mai 1712, da bei allen Untertanen der Cent Mergentheim keine Person sich an eine Hinrichtung außer der Rothaupt'schen erinnern könne, hielten diese das Zusammenrufen aller Centuntertanen zu einer Reparation für eine Novität. Die letzte Galgenrenovierung lag zu diesem Zeitpunkt schon über 80 Jahre zurück. Da das exakte Procedere einer Galgenreparierung nicht mehr bekannt war und auch keine Unterlagen darüber mehr aufgefunden wurden, suchte man Beispiele in der Literatur. Beigelegt in den Unterlagen des Stadtarchivs finden sich gedruckte Seiten, die eine Renovierung des Leipziger Galgens im Jahr 1706 schildern. Auf diese Vorgehensweise konnte man sich nun im weiteren Verlauf stützen. Zu diesem Zwecke erging am 4. Juni 1712 ein Regierungsdekret, das in allen Gemeinden der Cent Mergentheim verkündet wurde:

Am kommenden Montag, 6. Juni, hätten sich alle Vögte und Schultheißen sowie die Untertanen insgesamt, oder - wegen der Feldarbeit - eine Abordnung derselben, mit ihrem Ober- und Untergewehr ausgestattet einzufinden. In aller Frühe, wenn das Tor aufgesperrt würde, sollten sie auf dem Markt erscheinen und gewährtigen, waß ihnen weiters von hoher Cent Herrschaft wegen fürgetragen werden wirdt ${ }^{27}$.

Danach wurde die versammelte Centgemeinde mit fliegenden Fahnen und klingendem Spiel unter Anführung der Reiter, des Centgrafen (Gerichtsvorsitzenden) und der Gerichtsschöffen, von einer Eskorde begleitet, unter dem Spiel von sechs Pfeifern zum Hochgericht geführt. Abordnungen der Dörfer des Centbereichs (vom fränkischen Amt: Deubach, Sailtheim, Neubrunn, vom Odenwälder Amt: Ailringen, Rengershausen, Rot, Lustbronn, vom Nitzenhauser Amt: Nit-

26 StAL B 246 Bü 220.

27 StadtA Mergentheim F 3012. 
zenhausen, Berndshausen, Berndshofen) nahmen an der Prozession teil. Es folgten nach den Amtleuten die Zimmerleute mit ihren Äxten und Degen an der Seite, mit Bändern geschmückt. Drei Ellen lange Stangen, die mit Silber beschlagen waren, sowie geschmückte Fahnen führten diese mit sich. Jede Handwerkerkompanie (Maurer, Schlosser, Wagner ...) wurde begleitet von zwei Trommlern. Nun erfolgte das Ritual der Aufhebung der Anrüchigkeit. Der Ort wurde mit dreimaliger Wiederholung und Bekräftigung des Centgrafen ehrlich erklärt. Hierzu mussten alle sich in einem Kreis um das Hochgericht stellen. Ein Regierungsdekret wurde verlesen, das die Teilnahme und die Handanlegung keinem ahn seiner Ehr zum geringsten nachtheilig noch weniger schimpflich sein könne ${ }^{28}$.

Der Centgraf hatte mit einer Haue den ersten Hieb an dem Galgen ausgeführt. Darauf traten die Zimmerleute mit den Handlangern hervor, insgesamt 30 Mann, erledigten die Erdarbeiten und stellten das Gerüst auf. In viertelstündlichen Wechsel wurden die Handwerker ausgetauscht, damit eine möglichst große Zahl diese Arbeit verrichten musste unter der weiteren Anwesenheitspflicht aller. Durch diese Handanlegung war der gute Leumund aller Beteiligten gesichert. Immer wieder wird in diesem Zusammenhang von der Regierung betont, daß diese Handlung keinem weder iezt noch ins kunfftige zu einigem Nachtheil, Schimpf und Schaden gereichen sollte ${ }^{29}$.

Sollte dennoch einmal in diesem Zusammenhang einem Teilgenommenen ein Vorwurf gemacht, oder seine Ehre beschmutzt werden, sollte der Verleumder zu exemplarischer Strafe gezogen werden. Die neuen Balken wurden hinaufgezogen. Sie waren mit weiß verzinntem Blech beschlagen, damit das Holz nicht so schnell verrottete $^{30}$. Als zuletzt von den jüngeren Schmiedemeistern die Klammern eingeschlagen wurden, mit denen die Balken auf den steinernen Säulen befestigt waren, war die Arbeit gegen halb 12 Uhr mittags beendet. Daraufhin wurde das Zeichen zum Abmarsch gegeben und man zog wie man hinaus auch wieder in die Stad herein ${ }^{31}$. Soweit der Bericht des Centgrafen Wollenberger an die Regierung vom 12. Juni $1712^{32}$.

Die „einfachen“ Centuntertanen waren nun nach Hause entlassen. Die Vögte, Schultheißen und Handwerker hatten Anspruch auf Zehrungskosten, die besonders beim Straußenwirt in Rechnung gestellt wurden. 1716 beläuft sich die endgültige Kostenabrechnung für die Erneuerung des Hochgerichts von 1712 auf 78 fl 25 x. Davon entfielen allein fast $37 \mathrm{fl}$ auf die Rechnung des Straußenwirts für die Versorgung der Handwerker mit Speis und Trank. Diese exorbitant in die

28 Ebd.

29 StadtA Mergentheim F 3012, am 2. Juni 1712

30 Ebd., Brief der Stadtschreiberei vom 11. Mai 1712: wozu man 189 Plech dann bey 800 Plech Nägel nöthig hat.

31 StAL B 246 Bü 220.

32 David Christoph Wollenberger, Hofgerichtssekretär, Mitunterzeichner bei den städtischen Audienzprotokollen. 
Höhe geschossene Wirtshausrechnung blieb nicht unkommentiert. In einem Schreiben vom 16. Juli 1712 setzt sich Joh. Ignaz Hess ${ }^{33}$ mit dem Procedere der Handwerkerauswahl auseinander und bezieht sich dabei auf die Peinliche Halsgerichtsordnung Kaiser Karls V. Dort waren in $\S 215$ und $\S 217$ die Kosten dahingehend geregelt, dass die benötigte Anzahl der Handwerker durch Los bestimmt würde und diese entsprechend ihrem üblichen Lohn bezahlt würden ${ }^{34}$. Da neben der Carolina auch die Centordnungen und Artikelbücher, in denen der Rechtsgebrauch am jeweiligen Ort festgehalten war $^{35}$, in dem jeweiligen Gerichtsbezirks weiterhin Gültigkeit hatten, also nach ,,altem Herkommen“ der Rechtstraditionen vor Ort geurteilt und gehandelt wurde, sei der Brauch des Einkehrens rechtmäßig.

Der Stadtschreiber war nach Heranziehung dieser Stellungnahme von J. J. Hess auch der Meinung, dass alle Handwerker, auch die, die nur symbolisch Hand anlegen mussten, einen Anspruch auf Zehrungsgeld hätten, zumal der Centdistrikt nicht groß sei und die Anzahl der benötigten Handwerker oft kein Losverfahren nötig mache. Im Übrigen hätten auch die Vögte und Schultheißen der Ortschaften a bon conto dahier verzehrt was (leider?) den Centgrafen, den Schöffen und dem Gerichtsschreiber nicht gestattet sei, obwohl sie von Anfang bis Ende anwesend sein müssen und mit schwehren Pflichten sich belegen lassen müssen ${ }^{36}$. Gemeint sind hier unter anderem die zu vollziehenden Zeremonien, um die Anrüchigkeit des Hochgerichtes aufzuheben. Die Kosten lagen der Stadt schwer im Magen. Noch 1716 war nicht vollständig abgerechnet und viele Handwerker und Wirte warteten immer noch auf vollständige Begleichung ihres Arbeitslohnes sowie auf die Erstattung der Zeche ${ }^{37}$.

\section{Der Schnapp- oder Soldatengalgen}

An einer anderen markanten Stelle in Mergentheim, allerdings nicht in lichter Höhe, sondern in den Tauberauen der Arkau ${ }^{38}$, Richtung Igersheim gelegen, be-

33 StadtA Mergentheim F 3012. Johann Ignaz Hess war Ordenskanzler, † 1728. Sein Epitaph befindet sich im Mergentheimer Münster.

34 Vgl. Peinliche Halsgerichtsordnung (wie Anm. 25).

35 Weistümer: das durch Übung innerhalb einer Gemeinschaft entstandene Gewohnheitsrecht, in Satzungen festgehalten.

36 StadtA Mergentheim: 13. April 1712, Centgraf an Regierung.

37 StadtA Mergentheim F 3012, Specificatio deren bey der Centh Mergentheimb zu bezahlen stehenden Centkosten vom 5. Jun i 1716.

38 In der OAB Mergentheim von 1880, S. 434 steht: ,,der Bildstock beim Hofgarten, unweit der Tauber, gegen den Johannissteg, erinnert an den unglücklichen Sturz vom Pferde, den der damalige Commenthur zu Mergentheim Albrecht von Gebsattel im Jahr 1461 erlitt“, und der Chronist Breitenbach (wie Anm. 6, Bl.47v) schreibt von einem Johannissteg am Ende des Schlossgartens, auch Arkausteg genannt. Der Johannissteg war daher ungefähr auf Höhe des heutigen Verkehrserziehungsplatzes. 


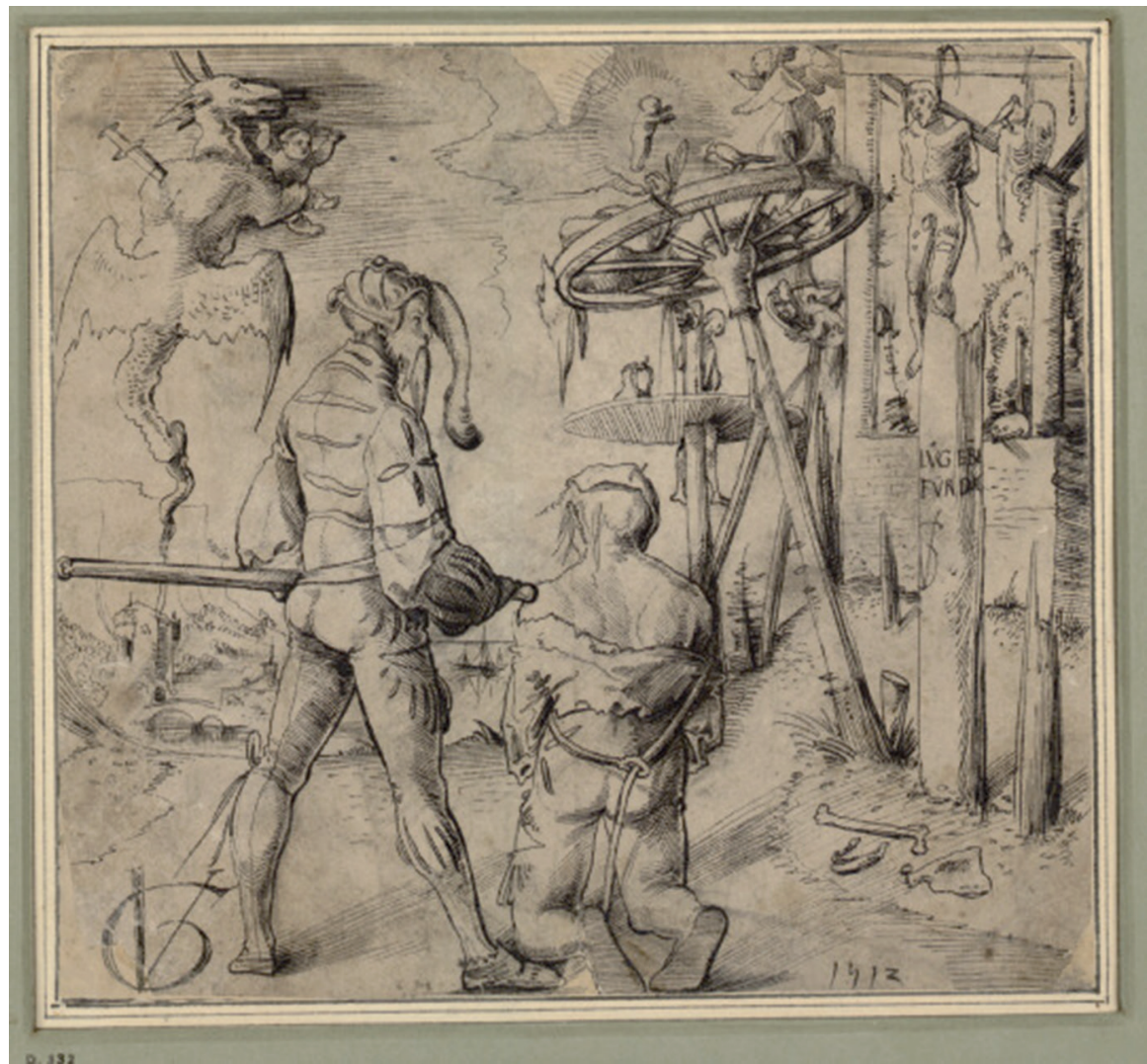

Abb. 4: Urs Graf d. ̈., Richtstätte, 1512, Federzeichnung auf bräunlichem Papier (Wien, Albertina)

fand sich eine andere Exekutionsstätte: Der Soldatengalgen. Sein Standort kann heute nicht exakt bestimmt werden, da nur die Angabe „beim Johannessteg“ überliefert ist. Dieser hölzerne Steg befand sich vor der Abzweigung des Mühlkanals, ungefähr auf Höhe des heute sich noch dort befindlichen Bildstocks. An einem solchen Soldatengalgen wurden die Strafen vor allem sinnbildlich vollzogen. Galgen, vor allem aber die Soldatengalgen, erfüllten nicht nur den Zweck, einen Delinquenten ins Jenseits zu befördern, sondern man konnte sie auch als eine Art schwarzes Brett betrachten. Hier wurde bekannt gemacht, welche Deserteure der Armee im Land umherstreiften oder wer steckbrieflich gesucht wurde. Eine solche Tafel wurde stellvertretend für den Entflohenen aufgehängt. Da dieser sich durch seine Fahnenflucht entzogen hatte, wurde stellvertretend für ihn als symbolischer Akt das Schild mit seinem Namen am Galgen befestigt. 


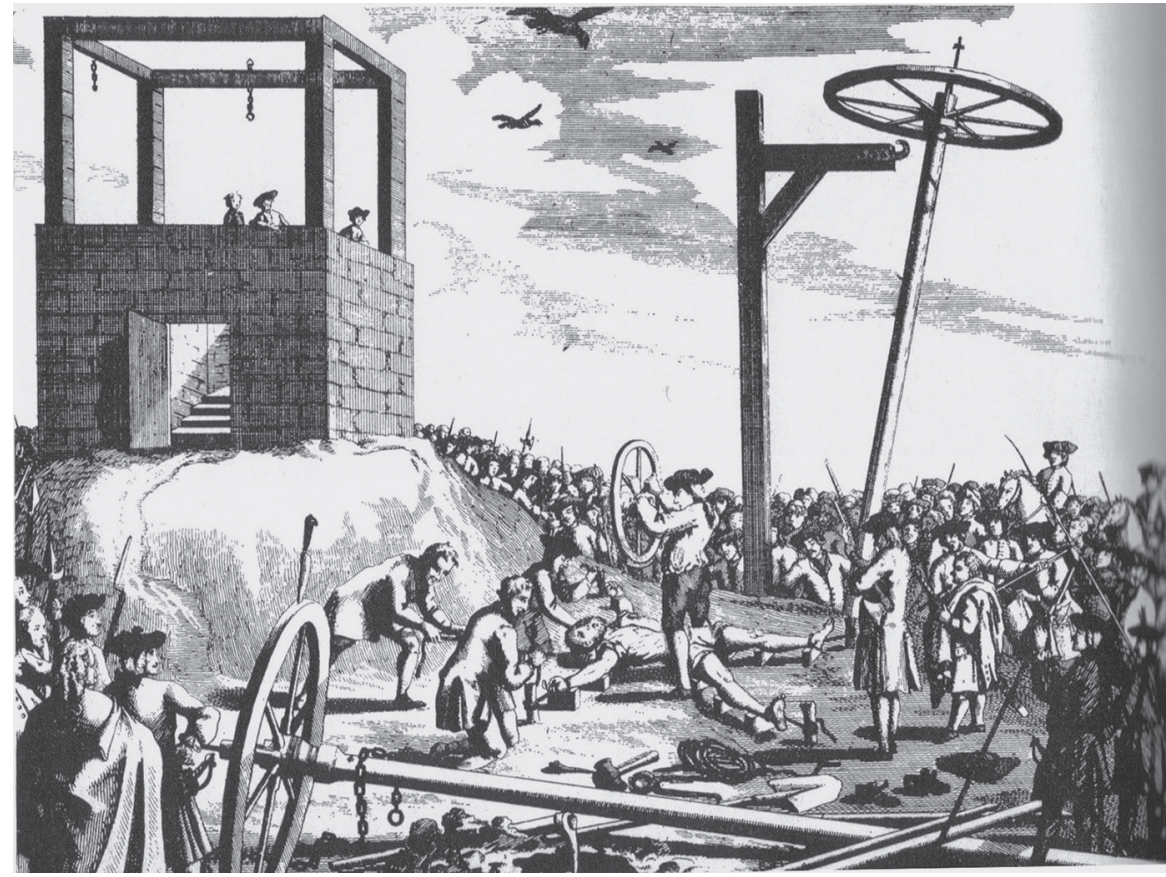

Abb. 5: Das Frankfurter Hochgericht 1741, Kupferstich

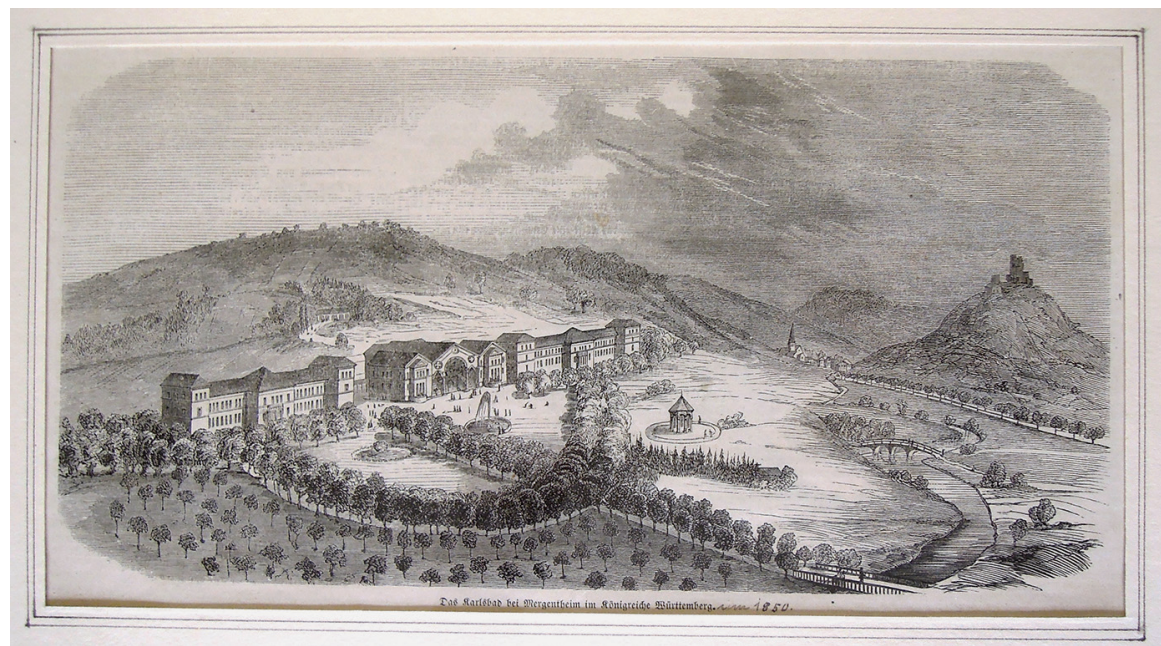

Abb. 6: Stahlstich um 1850, das Karlsbad bei Mergentheim (Deutschordensmuseum, Inv. Nr. 2529) 
Am 19. Februar 1710 erging ein Dekret der Mergentheimer Regierung, dass mit dem Anschlagen der Deserteurs Nahmen ahn Galgen wirklich - d.h. sofort fortzufahren sei und dazu ein neuer Galgen aufgerichtet werden sollte ${ }^{39}$. Manchmal - so in Frankfurt - standen solche Galgen auch beim Hochgericht und waren dazu da, die Soldaten zu „henken“ oder Leibesstrafen zu vollziehen. Diese wurden mit am Rücken gebundenen Händen hochgezogen und dann mit Schwung heruntergelassen, so dass die Arme ausgekugelt werden konnten ${ }^{40}$. Über eine solche Strafe ist jedoch in den Mergentheimer Unterlagen nichts zu finden. Die Jahre vor 1700 und danach waren geprägt durch Franzoseneinfälle und Truppendurchzüge durch die fränkischen Lande ${ }^{41}$. Dies waren die Folgen des Feldzuges der Franzosen in der Pfalz sowie später die Folgen des Spanischen Erbfolgekriegs zwischen Österreich und Frankreich. Dieser führte am 22. Juli 1707 ein französisches Streifcorps auch nach Mergentheim. In diesem Corps waren wahrscheinlich Ortskundige, denn sie stiegen nachts unweit des Hadergassentores, an einer Stelle, wo keine Wache stand und eine Mauerlücke vorhanden war, in den Zwinger ein und öffneten das Stadttor ${ }^{42}$. Ein Trupp drang bis zum Schloss vor und kidnappte den Statthalter, Hofrats-Präsident Friedrich Hermann von Kageneck, während die übrigen Soldaten die Stadt plünderten. Erst gegen eine Lösegeldzahlung wurde der Präsident Monate später von Straßburg wieder nach Mergentheim entlassen ${ }^{43}$.

Da man wahrscheinlich nicht ganz zu Unrecht vermutete, dass Deserteure aus den eigenen Reihen, die sich den Franzosen angeschlossen hatten, dieses hasardeurartige Eindringen in die Stadt erst möglich gemacht hatten, denn gute Ortskenntnis war dafür Voraussetzung, war man in der Folge - wegen des großen Schadens ${ }^{44}$ und vor allem wegen dieser ungeheuren Schmach - nicht gut auf Deserteure zu sprechen. Ein verschärftes Vorgehen gegen Deserteure war unumgänglich, und da man ihrer nicht habhaft werden konnte, heftete man ihre Na-

39 Nach StadtA Mergentheim F 3012 und StAL B 246 Bü 233 hier wird der alleinige Zweck für die Aufrichtung des Mergentheimer Soldatengalgens in der Anbringung dieser Schilder mit den Namen der Deserteure gesehen, Anordnung Veringens vom 19. Februar 1710.

40 Vgl. Krünitz (wie Anm. 1), Bd. 147, S. 428 sowie Johann Christoph Adelung: Grammatisch-kritisches Wörterbuch der hochdeutschen Mundart, Bd. 4, Wien, 1811, S. 129. Online Version: http:// lexika.digitale-sammlungen.de/adelung/online/angebot

41 In Mergentheim 1688 mit Brandschatzungen in vielen Dörfern um Mergentheim, siehe Breitenbach (wie Anm. 6), Bl. 109v.

42 Das Hadergassentor war eines der vier Stadttore. Es war der Stadteingang, den Reisende von Frankfurt oder Würzburg kommend und die Wolfgangsbrücke überquerend in die Stadt nahmen.

43 Breitenbach (wie Anm. 6), Bl. 113v und Heinrich Schmitt: Franzosen in Mergentheim 1704 und 1707. In: Veröffentlichungen des Altertumsvereins für württembergisch Franken 1895/96, S. 13-23, ebenso OAB Mergentheim 1880, S. 305, sowie Diehm (wie Anm. 5), S. 70 ff. Diehm gibt allerdings als Aufenthaltsort der Geisel Schöntal an. Ein Verzeichnis der durchgeführten Plünderungen und Misshandlungen, die während dieses Überfalls stattfanden, soll laut Diehm im StadtA vorliegen.

44 Breitenbach (wie Anm. 6): 88.000 fl für Lösegeld und Brandschatzung, vgl. auch OAB Mergentheim, S. 305, Verhör des Bürgermeisters, Stadtschreibers, Stadtlieutnants, Torwarts und Turmwächters, dessen Schilderung dort angeführt wird. 


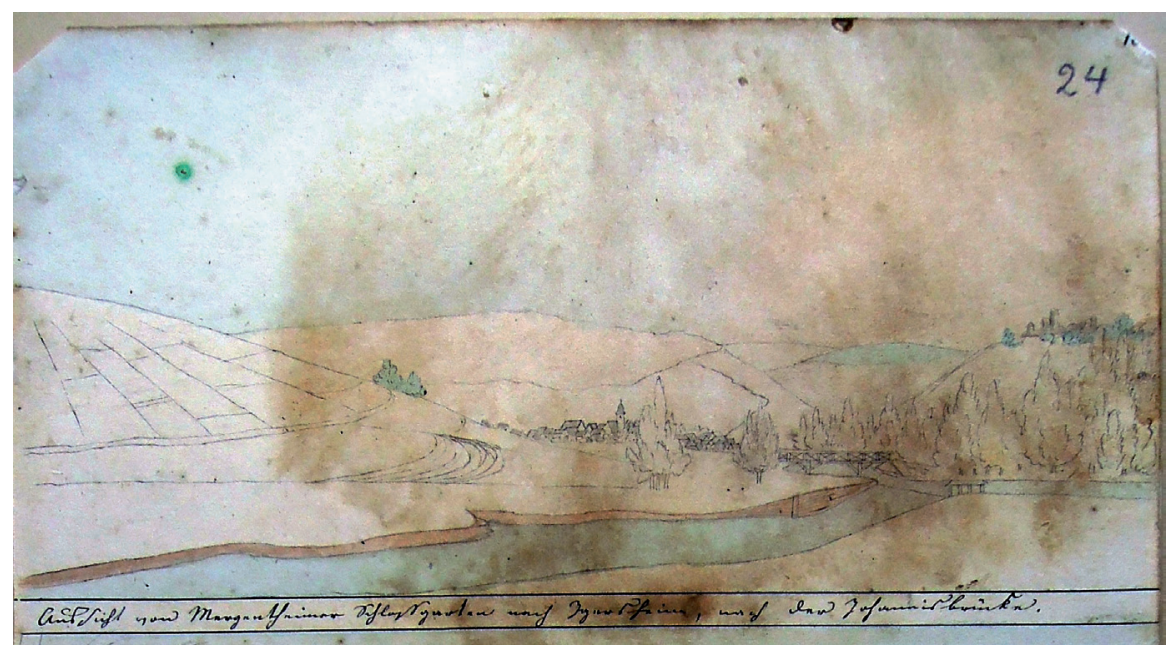

Abb. 7: (Georg?) Breitenbach, Aussicht von Mergentheim nach Igersheim mit der Johannisbrücke, kolorierte Federzeichnung, 1. Hälfte 19. Jahrhundert (Stadtarchiv Bad Mergentheim)

men an den Galgen. Neben der Anbringung der Schilder an solchen speziellen Soldatengalgen war es aber auch üblich, diese Namen am Hochgericht selbst anzubringen. In diesen unruhigen Zeiten waren auch die Schnappgalgen mit Namensschildern übersät. Von der Komturei erging der Befehl, unverzüglich mit der Neuaufrichtung eines Soldatengalgens zu beginnen weylen die neü ahngeworbene Recrouten noch alhier wahren ${ }^{45}$. Frühzeitig sollten sie zur Raison gebracht werden und die Funktion der Einschüchterung und Abschreckung nahm ein solcher Galgen durchaus ein.

In der Specification (Abrechnung) für die Neuaufrichtung fällt ein Posten besonders ins Auge: Dem Büttel wurden $1 \mathrm{fl} 15 \mathrm{x}$ gezahlt, dass er 54 Blech unter der Begleitung einer Eskorte und mit Trommelschlag öffentlich hinaus trug und diese dem Scharfrichter an der Richtstatt aushändigte ${ }^{46}$. Dieser wiederum schlug sie am neuen Galgen an, wofür er die stolze Summe von $9 \mathrm{fl} \mathrm{erhielt}^{47}$. Auf diesen blechernen Schildern waren jeweils die Namen der Deserteure aufgemalt. Im Februar 1710 wurde in dem Dekret zur Errichtung eines neuen Galgens besonders hervorgehoben, dass allen, die dort Hand anlegen, dieses an ihren Ehren

45 StAL B 246 Bü 233, zu diesem Zweck sollten die noch vorhandenen Schilder beim Abbruch des alten Galgens vom Scharfrichter geborgen und die noch lesbaren an die Herrschaft geliefert werden.

46 StAL B 246 Bü 233.

47 Ebd. laut Johann Gottfried Saulling, Johann Friedrich Veringen vom 3. März 1710. Nach Quittung von Scharfrichter Johann Simon Hofmann am 18.2.1710 erhielt er allerdings nur von Kammerrat Spang $6 \mathrm{fl}$. 
nicht im geringsten nachteilig sein könne und alle, die diese Handwerker durch Reden oder in irgend einer anderen Weise schmähten, mit scharfer Strafe von Amts wegen verfolgt werden sollten. Deutlich wird auch hier wieder, dass erst durch die Berührung des Scharfrichters dieser Galgen mit der Unehrenhaftigkeit belegt wurde, denn es wurde ausdrücklich betont, dass die Arbeiten der Handwerker „harmlos“ seien: Erst nach vollbrachter Arbeit solle der Scharfrichter selbigen berühren ${ }^{48}$.

Doch wie sah ein solcher Soldatengalgen aus? Die häufig nachweisbaren Reparaturen $^{49}$ sowie die Tatsache, dass er durch ein Tauberhochwasser weggespült werden konnte - so geschehen am Michaelstag (29. September) 1733 -, lassen die Vermutung zu, dass es sich hier um einen reinen Holzgalgen handelte. Auch die Handwerker, die an seinem Bau beteiligt waren, waren in erster Linie die Zimmerleute. Der Versuch, die Leinenweber als Handlanger heranzuziehen nach altem Herkommen und auch in Bayern auch so üblich - löste unter diesen einen Entrüstungssturm aus, dem mehrere Eingaben bei der Stadt und Regierung folgten. Trotz allem kamen die Leinenweber nicht umhin, drei ihrer Zunftgenossen als Handlanger für die Errichtung abzustellen ${ }^{50}$.

1748 machten die Zimmerleute wieder eine Zeche nach der Erneuerung des Schnapp- oder Soldatengalgens, die der Herrschaft bitter aufstieß. Eine kleine Bemerkung der Zimmerleute in dieser eingereichten Rechnung beschreibt die Form des Galgens, der darin als neuer Siebener bezeichnet wird ${ }^{51}$. Es handelte sich offensichtlich um eine in unserer heutigen Vorstellung übliche Galgenkonstruktion: Ein senkrechter Balken, daran oben ein waagrechtes Balkenstück, im Winkel verstärkt durch eine Strebe. Dies ergibt in der Form die Zahl 7.

\section{Der letzte Einsatz am Hochgericht}

Obwohl nach einer königlich württembergischen Verordnung vom 27. April $1811^{52}$ alle an den Straßen stehenden Galgen abgebrochen werden sollten, hat sich das Hochgericht von Mergentheim noch einige Jahre gehalten. Die letzten Hinrichtungen an diesem Ort fanden im Jahr 1818 statt, als der Mordbrenner

48 StadtA Mergentheim F 3012.

49 StAL B 246 Bü. 233: In einer Abrechnung vom 14. Dezember 1733 werden dem Scharfrichter Johann Simon Hofmann 3 fl 15 x bezahlt, da er die alten Bleche abmachte und die alten Gerichtsbalken hinwegführte.

50 StAL B 246 Bü 220: Bericht Molitors, auch Krünitz (wie Anm. 1) bezieht sich darauf und stützt sich auf Wiguleus Hund: Bayrisches Stammenbuch [...]. Ingolstadt 1598.

51 StAL B 246 Bü 233, Abrechnung vom 24. Februar 1748 über die Verzehrkosten beim StrauBenwirt von $15 \mathrm{fl} 20$ x. Der Name Siebener scheint nicht ungebräuchlich gewesen zu sein. In der Provinz Utrecht ist der Name Siebenbäumchen für einen Hügel überliefert, allerdings befand sich dort das Rad. Es könnte auch ein Hinweis auf einen solchen Holzgalgen sein. Siehe dazu Jost Auler: Richtstättenarchäologie in den Niederlanden. Das Beispiel Amersfoort (Provinz Utrecht), in: Jost Auler (Hg.): Richtstättenarchäologie 2. Dormagen 2010, S. 30-33.

52 HStAS E 302 Bü 782. 
Michael Singer aus Zaisenhausen und der Elternmörder Johann Georg Dietrich aus Unterhöfen bei Öhringen im Juli und September ihren letzten Gang zum Hochgericht antreten mussten ${ }^{53}$. Ein Augenzeugenbericht ist durch die Schellsche Chronik überliefert. Schell hat in seiner Jugend diese Exekution miterlebt ${ }^{54}$. Wahrscheinlich musste er als einer der Schüler, die zur Teilnahme verpflichtet waren, und die vielfach - wie auch aus anderen Orten überliefert - als Chorsänger in dem streng geregelten Procedere einer Hinrichtung ihre Rolle einnehmen mussten, den Gang der Dinge verfolgen. Michel Singer aus Zaisenhausen wurde im Juli wegen Raubmord und Brandstiftung zum Tode durch das Schwert mit anschließender Räderung verurteilt. Üblicherweise kniete der Delinquent, während der Scharfrichter von hinten in waagrechtem Streich den Kopf vom Rumpf trennte (s. Abb. 4). In Mergentheim war, wie in vielen anderen Orten auch, seit vielen Jahrhunderten ein Stuhl gebräuchlich ${ }^{55}$, auf dem der Delinquent festgebunden wurde. Erst nach der Enthauptung erfolgte die Räderung am toten Körper ${ }^{56}$. Die Räderung wurde hier am bereits Hingerichteten vollzogen. Daher war es nicht das Ziel, die Grausamkeit der Hinrichtung für den Delinquenten zu erhöhen, sondern die Schwere des Verbrechens sollte, widergespiegelt in der Hinrichtungsart, dargestellt werden. Es war ein besonderer Gnadenerweis des Gerichtes, wenn der Delinquent einen schnellen Tod durch das Schwert zugestanden bekam. Anders verhielt es sich bei der Exekution von Johann Georg Dietrich, der auf einer rohen Kuhhaut zur Hinrichtungsstätte geschleift und dort gerädert wurde ohne zuvor zum Schwert begnadigt worden zu sein. Dass im Jahr 1818 die außerordentlich grausame Art der Räderung nochmals durchgeführt wurde ist ungewöhnlich und spricht für die Schwere des Verbrechens. Die - soweit bekannt - letzte Räderung in Deutschland fand 1852 in Preußen statt, die letzte in Württemberg $1819^{57}$.

Wie schon bei der Reparatur des Hochgerichtes war auch bei den Hinrichtungen die Anwesenheit der Bevölkerung Pflicht. Darüber hinaus gestalteten sich allerdings die Hinrichtungen zu großen Menschenaufläufen, denn auch aus der weiteren Umgebung kamen Sensationstouristen angereist, um sich dieses Spektakel als Abschreckung dienen zu lassen. Die Stadt musste sich entsprechend wappnen und zusätzliche Polizei und weitere Hilfskräfte anstellen. Nach zeitgenössischen Berichten sollen sich in Mergentheim 8000 Menschen zu diesem Ereignis zusammengefunden haben.

53 Vgl. zu beiden: Fritz (wie Anm. 1), S. 265.

54 Johann Schell, Kaufmann und Stadtrat in Mergentheim, 1802-1861. Sein Familiengrab ist noch auf dem alten Friedhof in Mergentheim zu finden

55 Breitenbach (wie Anm. 6), Bl. 14v nennt schon in einer Abrechnung der Hexenprozesse einen rot angestrichenen Stuhl, Hexenprozesse.

56 Sein Augenzeugenbericht ist abgedruckt bei Alice Ehrmann-Pösch: Der letzte Scharfrichter von Mergentheim. In: WFr 95 (2011), S. 149-178.

57 Fritz (wie Anm. 1), S. 265-271 mit einer Übersicht über die Todesurteile in Württemberg zwischen 1818 und 1871. Zur letzten Räderung in Preußen ebd., S. $251 \mathrm{f}$. 
1818 waren öffentliche Hinrichtungen nichts Alltägliches mehr, denn die Hinrichtungszahlen nahmen schon seit der Mitte des 18. Jahrhunderts immer mehr ab. Seit der Mitte des 19. Jahrhunderts fanden sie unter Ausschluss der breiten Öffentlichkeit hinter den Gefängnismauern statt. ${ }^{58} \mathrm{Zu}$ Zeiten der öffentlich vollzogenen Hinrichtungen waren die häufigsten Arten der Hinrichtung die Enthauptung und das Aufhängen am Galgen. Die Enthauptung musste nicht unbedingt am Hochgericht erfolgen. Bezeugt sind in Mergentheim auch Enthauptungen auf dem Marktplatz ${ }^{59}$, sowie auf dem Tauberwasen bei der Wolfgangsbrücke ${ }^{60}$. Räderungen stellten mit die grausamste Art der Hinrichtung dar. In Mergentheim sind solche zwar bezeugt, doch sind sie die absoluten Ausnahmen. Wie die Quellen vermitteln, war nicht nur der Galgen am Hochgericht aufgebaut, sondern auch ein Schafott, auch Blutgerüst genannt: eine Art Bühne, oft nur eine Plattform aus mehreren Brettern zusammengenagelt, auf der die Decaption oder auch Decollierung (= Enthauptung) vorgenommen wurde. Eine fest gemauerte „Köpfstätte“ oder „Rabenstein“ war in Mergentheim nicht vorhanden, wie auch schon Franz Simon Molitor in seinem Bericht 1712 schildert $^{61}$, ebenso wenig eine Mauer, die diesen Bezirk vor ungewolltem Zutritt verschloss.

\section{Was geschah mit den Toten am Hochgericht?}

Die Vielfalt der unterschiedlichen Hinrichtungsarten lässt schon erahnen, dass auch der tote Körper gewisse Funktionen zu erfüllen hatte. Es darf hierbei auch nie außer Acht gelassen werden, dass ein Galgenbezirk mit „Gebrauchsspuren“ auch weithin sichtbar die Herrschaftsausübung dokumentierte. Abschreckung und Respekteinflößung sind natürlich bei einem in Anspruch genommenen Galgen wesentlich effektiver. Für einen Hingerichteten bedeutete das im schlimmsten Fall, dass sein Leichnam Wind und Wetter ausgesetzt blieb, bis der natürliche Verfall oder der Appetit der Galgenvögel ihn vom Strick, bzw. von der Galgenkette erlöste. Das Hängenlassen der Leiche am Galgen galt Strafverschärfung, die Beerdigung der Leiche als Strafmilderung. Die Mergentheimer Obrigkeit war in aller Regel eher gnädig, denn in den Abrechnungen der Hinrichtungen wird häufig der Totengräber mit einem geringen Salär entlohnt, um den armen Sünder zu begraben.

58 Ebd., S. 265. Zur Entwicklung im 18. Jahrhundert: Ders.: Herzog Carl Eugen, König Friedrich und die Abschaffung der Folter in Württemberg. In: ZWLG 67 (2008), S. 183-248.

59 Diehm (wie Anm. 5), S. 57. Die Hinrichtungen der Rädelsführer vom Bauernaufstand 1525, die von Mergentheim waren, fanden zum Teil auf dem Marktplatz statt.

60 StadtA Mergentheim F 3005: Vgl. dort die Scheinhinrichtung von Barthel Hänfling von 1682.

61 StAL B 246 Bü 220, Bericht vom 20. Mai 1712: undt obschon dahier zu Mergentheim kein dergleichen Rabenstein befindlich, daß Hochgericht auch weder mit einer Mauren umbfangen, oder durch eine Thüre beschlossen ist. 
Keineswegs gnädig verfuhr der württembergische König Friedrich 1809 bei der Hinrichtung von Franz Werner, einem Anführer des Mergentheimer Aufstandes, bei dem sich die Ordensuntertanen gegen die württembergische Okkupation zur Wehr gesetzt hatten. Nach einem kurzen Martialgericht (= Standgericht) wurde er noch am selben Tag aufgehängt. Die Leiche musste am Galgen verweilen, was die Schwere des Delikts - Rebellion gegen die neue württembergische Landesherrschaft - unterstreichen sollte. Erst nach vier Wochen erging der Gnadenerweis des Königs, die Leiche vom Holz abzunehmen ${ }^{62}$.

In der Centtaxordnung (= Gebührenordnung für das Gerichtswesen) Clemens Augusts von 1732, erlassen für das gesamte Meistertum um der großen Ungleichheit und mannigfältigen Excessen Einhalt zu gebieten, wird der Totengräber bezahlt mit 1 fl 30 x, der Schreiner für die Totenlade mit 1 fl 20 x, der Fuhrmann, der den toten Delinquenten zum Kirchhof fährt, mit $1 \mathrm{fl}^{63}$.

Ein christliches Begräbnis für einen Hingerichteten bezeugt ein gewisses Maß an Nächstenliebe. Aber er wurde nicht durch das gewöhnliche Friedhofstor zu der Begräbnisstätte gebracht, sondern durch einen kleinen Seiteneingang in der Friedhofsmauer. Nach Breitenbach befand sich an der Westseite des Friedhofes ein breites hohes Tor und von diesem einige Schritte abwärts war für arme Sünder ein kaum 6 Fuß hoher und 3 Fuß breiter Eingang vorhanden, der allerdings schon seit Beginn des 19. Jahrhunderts nicht mehr geöffnet ist ${ }^{64}$. Neben dem Totengräber besorgte auch der Scharfrichter diese Aufgabe. In der Centtaxordnung von 1732 war er für diese Arbeit vorgesehen: Der Fuhrlohn belief sich auf $1 \mathrm{fl}$, der Grabaushub auf 40 x. Des Weiteren taucht in dieser Ordnung noch ein zusätzlicher Posten diesbezüglich auf: für das Grab unter dem Galgen oder sonsten zu machen $1 \mathrm{fl}$.

Viele Galgenplätze, so auch der Mergentheimer, dienten nicht nur als Hinrichtungsstätte, sondern waren zugleich auch Begräbnissorte. Unter dem Galgen vom Scharfrichter oder seinen Henkersknechten verscharrt zu werden, war eine Form der Strafverschärfung, denn man war nicht in geweihter Erde bestattet, sondern man war gebannt an diesen friedlosen Ort. In den Malefizkosten des Buchener Statutenbuch von 1723 wird das außergewöhnliche Zugeständnis einer Bestattung in geweihter Erde schon an der Formulierung deutlich: so einer mit dem Schwerdt hingerichtet und zu christlichem Begräbnus begnadet wirdt ${ }^{65}$. Bei den Ausgrabungen am Ellwanger Galgen 1991 traten etliche menschliche Überreste, die in relativ flache Gruben hineingeworfen und -gezogen wurden, zu Tage, daneben auch tierische Skelettfunde. Weder ein Sarg noch eine würdige Bettung, sondern ein schnelles Verscharren war hier die Regel ${ }^{66}$.

62 Hartwig Behr: Todesurteile und Festungshaft, der König nimmt brutal Rache. In: Tauber-Zeitung 20. Juni 2009.

63 StadtA Mergentheim F 3011.

64 Breitenbach (wie Anm. 6), B1. 29r.

65 StadtA Mergentheim F 3011.

66 Piech (wie Anm. 23). 


\begin{abstract}
Aberglauben und Magie
Nur kurz angesprochen sei hier das weite Feld des Aberglaubens und der Magie, das sich rund um die Galgenplätze auftut. Die Leichen der Erhängten, die unbewacht am Galgen hingen, waren begehrte Objekte von Quacksalbern, Amuletthändlern, Dieben und vollkommen legal auch vom Scharfrichter. Angefangen von der Kleidung, wurden auch Teile des Körpers verwertet, besonders die Daumen waren wertvolle Verkaufsartikel, denn als Diebsdaumen bei sich getragen, verhinderten sie die Entdeckung einer Straftat, oder halfen gegen Warzen und Zauberei ${ }^{67}$. Hautstücke waren beliebte Amulette zur Abwehr von allerlei Übel. Der Fantasie waren hier keine Grenzen gesetzt.

Der Galgen selbst war ebenfalls Objekt der Begierde für die Schwarzkünstler. Galgenspäne wurden teuer gehandelt. Ein Ring, geschmiedet aus der Galgenkette, löst Krämpfe ${ }^{68}$, daraus hergestellte Sporen für die Pferde sollten bewirken, dass sie hurtig fortgehen sollen ${ }^{69}$, auch sogenannte Freikugeln wurden durch die Hirnschalen Gehängter gegossen und aus dem Metall vom Galgenketten hergestellt. Unter dem Galgen wächst auch, wie immer wieder betont wurde, die zauberwirksame Alraune (auch genannt Mandragora) besonders gut, da sie sich von den abgehenden Körpersäften der Erhängten nährt. Die Form ihrer Wurzel ist menschenähnlich und so bekam die Wurzel auch den Namen Galgenmännchen. Dieses Nachtschattengewächs gilt seit der Antike als Zaubermittel.

Auch die offizielle Medizin war dankbar über Studienmaterial. Seit dem 18. Jahrhundert waren die Scharfrichter in Universitätsstädten verpflichtet die Leichen der Gehängten an die Anatomie der medizinischen Fakultäten abzuliefern, in honorem academiae wie das Lexikon von Krünitz im 18. Jahrhunderts beschreibt $^{70}$.
\end{abstract}

\title{
Das weitere Schicksal des Mergentheimer Hochgerichts
}

Das Mergentheimer Hochgericht war, wie gezeigt wurde, nicht nur der Platz des Galgens, sondern hier wurden auch Hinrichtungen mit dem Schwert sowie Räderungen ausgeführt. Daher brauchte man ein Schafott, eine Hinrichtungsbühne, die allerdings nicht wie in anderen Orten üblich aus Stein ausgeführt wurde, sondern wohl eher ein zur jeweiligen Hinrichtung wieder neu auszuführendes Brettergerüst war. Die Materiallieferungen der Müller 1711 legen den Schluss

67 Richard Evans: Rituale der Vergeltung. Die Todesstrafe in der deutschen Geschichte 15321987. Berlin 2001, S. 129.

68 Johann Theodor Jablonski: Allgemeines Lexikon der Künste und Wissenschaften. Leipzig 1721, S. 360.

69 Krünitz (wie Anm. 1), S. 681

70 Ebd. und HStAS E 302 Bü 785. 
nahe, dass ein kleiner Sockel aus Stein gesetzt und darauf Bretter gelegt wurden (Beschreibung siehe oben).

Der Mergentheimer Galgen stand nach 1818 viele Jahre verwaist und wurde um 1850 eingeebnet. Nach alten Erzählungen und Erinnerungen kamen die steinernen Trommeln der Pfeiler in den Garten des Kapuzinerklosters ${ }^{71}$, wo sie noch viele Jahrzehnte lagen. Um 1900 waren auf dem Kitzberg am Galgen die Fundamentgruben der steinernen Säulen noch deutlich erkennbar ${ }^{72}$. Von nahezu allen diesen Stätten hat sich nichts erhalten, zumindest nicht über der Erde. Als Bodendenkmäler werden Galgenplätze seit der 2. Hälfte des 20. Jahrhunderts verstärkt beachtet. Es entwickelt sich seit ein paar Jahrzehnten ein Zweigfach der Archäologie: die Richtstättenarchäologie ${ }^{73}$. Diese Orte werden ergraben, um mittelalterliche und neuzeitliche Zeugnisse der juristischen Exekutive zu sichern. Richtstätten als kulturhistorische Zeugnisse der Strafrechtspflege, aber auch als Bestattungsorte, an denen mannigfaltige Skelettreste geborgen werden, lohnen der genaueren Untersuchung, wie die Ellwanger Ausgrabung aus dem Jahr 1991 zeigt.

Auf keinen Fall sollte man achtlos im Rahmen von Überbauungen, oder Erdaushebungen unwissend oder kalkulierend die Überreste beseitigen, sondern respektvoll, verantwortungsbewusst und kulturwissenschaftlich exakt damit umgehen, auch um mehr Aufschluß über die Hinrichtungspraxis in der Cent Mergentheim zu erfahren. Bisher sprechen nur die schriftlichen Quellen, oder Denkmale wie die an manchen Orten noch anzutreffenden Sühnekreuze von vergangener Rechtssprechungspraxis.

Hochgerichte sind also Orte, die einerseits die Souveränität eines Herrschaftsgebietes darstellen und gehegte Rechtsbezirke. Andererseits sind es Orte des menschlichen Dramas, das in vielerlei Akten einem bewegten Publikum vorgeführt und vollzogen wurde, gleichsam als Spektakel der Abschreckung und idealer Weise als Opfergang eines reuigen Sünders ${ }^{74}$. Es sind aber auch Orte des Grauens, an dem Widergänger und ähnliches (Galgenweible) ihr Unwesen treiben sollen und eine magisch aufgeladene Sphäre dem Wunderglauben an ,Reliquien"Vorschub leistet. Und es sind trotz allem aber auch Begräbnisplätze, auch wenn sie keine christlich geweihten Bezirke sind.

71 Im 19. Jahrhundert war die Stadtschäferei dort untergebracht.

72 StadtA Mergentheim Rep. 245e, Schmittsche Aufzeichnungen.

73 Vgl. dazu die Untersuchungen von Piech (wie Anm. 23) über den Ellwanger Galgen aus dem Jahr 2009, sowie Jost Auler: Richtstättenarchäologie in der Schweiz. Ein Überblick. In: Archäologisches Korrespondenzblatt 37 (2007), S. 297-312.

74 Vgl. dazu Richard van Dülmen: Theater des Schreckens. Gerichtspraxis und Strafrituale in der frühen Neuzeit. München ${ }^{4} 1995$, S. 161 ff., in dem er die Hinrichtungsrituale als eine zeremonielle Handlung betrachtet, die an liturgische-religiöse Vorstellungen anknüpft. 
